ISSN 0103-5150

Fisioter. Mov., Curitiba, v. 24, n. 4, p. 673-682, out./dez. 2011

Licenciado sob uma Licença Creative Commons

\title{
Variáveis hemodinâmicas, hemogasométricas e respiratórias em pacientes cardiopatas submetidos ao teste de respiração espontânea
}

\author{
Hemodynamic, arterial blood gases and respiratory variables in \\ cardiac diseases patients undergoing spontaneous breathing test
}

\author{
Jaqueline Fernandes Cruz Rolim ${ }^{[a]}$, Nelson Henrique Lopes de Moraes ${ }^{[b]}$, José Ribeiro Uchôa Junior ${ }^{[c]}$ \\ [a] Graduanda do Curso de Fisioterapia da Universidade Católica de Pernambuco (Unicap), Brasil, Recife - PE, e-mail: \\ rolim.jack@gmail.com \\ [b] Especialista em Fisioterapia Cardiorrespiratória, professor do Centro de Ciências Biológicas e Saúde (CCBS) da Unicap, \\ orientador, fisioterapeuta, Recife, PE - Brasil, e-mail: nhenrique@oi.com.br \\ [c] Especialista em Terapia Intensiva pela Associação Brasileira de Fisioterapia Cardiorrespiratória, Fisioterapia em Terapia \\ Intensiva (Assobrafir), orientador, Recife, PE - Brasil, e-mail: jruchoajr@gmail.com
}

\section{Resumo}

Contextualização: Apesar de existirem situações em que há necessidade da ventilação mecânica (AVM), sua retirada (desmame) deve ocorrer tão breve quanto possível, sendo o teste de respiração espontânea (TRE) uma ferramenta útil para abreviar e reduzir os riscos. Existem diversas formas de realizar o TRE, porém, em pacientes cardiopatas, o mais eficaz parece ser associado à pressão de suporte (PSV) e à pressão positiva no final da expiração (PEEP). Objetivo: Analisar, ao início e ao término do TRE no modo PSV, o comportamento das variáveis hemodinâmicas, hemogasométricas e respiratórias em pacientes cardiopatas. Método: 22 pacientes cardiopatas foram submetidos ao TRE por 30 minutos, dos quais cinco foram excluídos e 17 conseguiram concluir o teste. Para mensuração da força muscular inspiratória e da mecânica respiratória, avaliou-se imediatamente antes do TRE: pressão inspiratória inicial e inspiratória máxima, resistência das vias aéreas, complacência estática e dinâmica seguida da avaliação do volume corrente, frequência respiratória, parâmetros hemodinâmicos e hemogasométricos. Todos os parâmetros foram reavaliados 30 minutos após o TRE. Resultados: Os valores tratados na análise estatística dessas variáveis não apresentaram diferença significativa, apenas o índice de desmame ventilatório mostrou variação significativa $(p=0,011)$. Conclusão: Por meio do presente estudo, percebeu-se que a maior parte dos pacientes cardiopatas submetidos ao TRE utilizando PSV manteve-se em estabilidade hemodinâmica, hemogasométrica e respiratória. Além disso, percebeu-se que cerca de $82 \%$ obteve 
sucesso ao desmame, sugerindo que PSV é um método seguro e eficaz na interrupção AVM. Contudo, por causa da amostra reduzida, o desenvolvimento de estudos semelhantes torna-se necessário.

Palavras-chave: Desmame. Ventilação mecânica. Avaliação.

\section{Abstract}

Background: Although there are situations requiring mechanical ventilation (AVM), withdrawal (weaning) should occur as soon as possible, and the spontaneous breathing test (SBT) is a useful tool to shorten and reduce the risks. There are many ways to implement the SBT, however, in patients with heart disease, the most effective one seems to be associated with pressure support (PSV) and positive pressure at the end the expiration (PEEP). Objective: To analyze, at the beginning and the end of the SBT in the PSV mode, the behavior of hemodynamic, respiratory and blood gas variables in patients with heart disease. Methods: 22 cardiac patients underwent to SBT for 30 minutes, of which five were excluded and 17 were able to complete the test. For measurement of inspiratory muscle strength and respiratory mechanics, it was evaluated immediately before SBT: initial inspiratory pressure and maximal inspiratory, airway resistance, static compliance and dynamic, then the assessment of tidal volume, respiratory rate, arterial blood gases and hemodynamics parameters. All parameters were reassessed at the time 30 minutes after the SBT. Results: The values treated in the statistical analysis of these variables showed no significant difference, only the index of weaning showed significant variation ( $p=0.011)$. Conclusion: Through this study, it was noted that most cardiac patients undergoing to SBT using PSV remained in stable hemodynamic, respiratory and blood gas. Moreover, it was noticed that about $82 \%$ were successful at weaning, suggesting that PSV is a safe and effective method in AVM interruption. However, due to small sample size the development of similar studies is needed.

Keywords: Weaning. Mechanical ventilation. Assessment.

\section{Introdução}

Denomina-se ventilação o processo cíclico de condução do ar da atmosfera até os alvéolos pulmonares, onde o pulmão recebe um determinado volume de ar e o elimina na forma de gás (1). Em indivíduos saudáveis, esse processo é realizado pelos músculos inspiratórios de forma ativa e de forma passiva pelo recuo elástico do pulmão e da parede torácica (2). Porém, em situações em que há impossibilidade dessa manutenção, a assistência ventilatória mecânica (AVM) torna-se necessária, sendo essa definida como um suporte ventilatório artificial (3-5).

Entre os pacientes que evoluem com a utilização deste recurso, necessitando dele, os pacientes cardiopatas apresentam características peculiares, entre elas: aumento do trabalho respiratório decorrente da redução da complacência pulmonar causada pelo edema pulmonar; aumento da resistência das vias aéreas em virtude do surgimento do edema peribrônquico; piora da troca gasosa pelo aumento da barreira alvéolo capilar, aliado ao aumento da sobrecarga cardíaca e redução da oferta de oxigênio aos tecidos (6).
Grande parte desses pacientes, além dos problemas supracitados, é submetida a procedimentos cirúrgicos para revascularização miocárdica, que, na maioria dos casos, são realizados mediante esternotomia com abertura do tórax, comprometendo, dessa forma, a complacência torácica, bem como levando a complicações como dor, derrame pleural e atelectasia. Esses fatores contribuem para maior tempo de AVM e consequente redução da força muscular inspiratória, o que dificulta ainda mais a retirada deles do suporte ventilatório mecânico (7-10).

Essa retirada ou interrupção do suporte ventilatório deve ser realizada o mais precocemente possível, uma vez que seu emprego por longos períodos pode acarretar complicações como infecções, barotraumas, fraqueza e incoordenação dos músculos respiratórios. 0 desmame é definido como uma mudança da ventilação mecânica para a espontânea, havendo, porém, variação na forma de administrar esse processo, o qual pode ser classificado como simples, difícil ou prolongado. Essa classificação gera maior complexidade e dificuldade na previsão do sucesso, 
sendo, portanto, imprescindível a disponibilização de recursos que possibilitem subsídios para melhor predizer o sucesso ao desmame e reduzir os riscos intrínsecos ao mesmo (11-14).

Nesse aspecto, surge como fator, corriqueiramente utilizado para abreviar o desmame e reduzir os seus riscos, o teste de respiração espontânea (TRE), que consiste na manutenção do paciente respirando espontaneamente por meio do tubo $t$, com suporte pressórico mínimo ou ainda por meio da manutenção de uma pressão positiva contínua das vias aéreas (do inglês CPAP. Para os pacientes cardiopatas, o TRE realizado com pressão de suporte ventilatório (PSV) associado à pressão positiva contínua no final da expiração (PEEP) parece ser o método mais eficaz (15).

Diante do exposto, o objetivo deste estudo é analisar, ao início e ao término do TRE no modo PSV, o comportamento das variáveis hemodinâmicas, hemogasométricas e respiratórias em pacientes cardiopatas.

\section{Materiais e métodos}

Trata-se de um ensaio clínico, quali-quantitativo, vinculado ao Hospital Geral Otávio de Freitas com CAAE n. 5035.0.000.344-09, realizado nas unidades coronarianas I e II do Pronto Socorro Cardiológico de Pernambuco (Procape).

Fizeram parte desse estudo 22 pacientes cardiopatas, de ambos os gêneros, submetidos à AVM de forma invasiva por período $\geq 24$ horas, no modo PSV, escala de coma de Glasglow $\geq 9(16,17)$, escala de sedação de Ramsay (18) entre 2 e 3, estabilidade hemogasométrica e hemodinâmica e pressão inspiratória máxima $\left(\mathrm{PI}_{\text {MÁX }}\right) \leq-25 \mathrm{cmH}_{2} \mathrm{O}$.

Foram excluídos ou tiveram procedimento interrompido aqueles pacientes que apresentaram alterações relacionadas à variação da pressão arterial sistólica (PAS) $\geq 180 \mathrm{mmHg}$, pressão arterial diastólica (PAD) $\geq 100 \mathrm{mmHg}$, frequência respiratória (FR) $>35 \mathrm{ipm}$, volume corrente (VC) $<5 \mathrm{ml} / \mathrm{kg}$, saturação periférica de oxigênio $\left(\mathrm{SpO}_{2}\right)<90 \%$, sudorese, cianose, utilização da musculatura acessória da respiração e batimentos da asa do nariz. Dessa forma, cinco pacientes foram excluídos por apresentarem falha antes de concluírem os 30 minutos do TRE, sendo dois por apresentarem rolha no tubo endotraqueal, dois por rebaixamento do nível de consciência e um pela diminuição do VC e aumento da FR.
A amostra final foi constituída por 17 pacientes, 10 (58,8\%) gênero feminino e 7 (41,2\%) gênero masculino, com idade média $62,65 \pm 11,5$ anos, altura média de 1,56 \pm 0,09 metros, cuja condição patológica foi: infarto agudo do miocárdio anterior/choque cardiogênico 4 (23,5\%), síndrome coronariana aguda $4(23,5 \%)$, insuficiência cardíaca congestiva (ICC)/miocardiopatia dilatada 8 (47,1\%), estenose de mitral 1 (5,9\%).

Após análise dos parâmetros hemodinâmicos, hemogasométricos e respiratórios, aqueles que preencheram os critérios de inclusão foram submetidos ao TRE. A partir desse momento, foram posicionados em decúbito supino elevado a $45^{\circ}$, angulação esta mensurada com a utilização de um goniômetro universal (CARCI-São Paulo-Brasil) e mantida durante todo período do estudo.

A estatura dos pacientes foi obtida por meio da mensuração da semienvergadura, utilizando a régua antropométrica de madeira de um metro (CARCISão Paulo-Brasil), tendo como ponto de referência o osso esterno até a falange distal do dedo médio, obtida com abdução de $90^{\circ}$ do ombro; posteriormente, era alcançado o produto do resultado da semienvergadura por dois (19).

Após obtenção da estatura, foi calculado o peso ideal por meio da equação do peso ideal de Massachustes (20): 50 + 0.91x (altura em cm - 152.4) e 45.5 + 0.91x (altura em $\mathrm{cm}-152.4$ ) para o gênero masculino e feminino, respectivamente. Dando sequência ao estudo, foi calculado o VC ideal, utilizando-se o valor de $8 \mathrm{ml} / \mathrm{kg}$, o qual foi utilizado para a mensuração da mecânica respiratória.

A mensuração da mecânica respiratória foi obtida com pressão do cuff mantida em $30 \mathrm{cmH}_{2} \mathrm{O}(21,22)$ (Marce-cuff pressure cauge, VBM - Alemanha), sem presença de escape aéreo, no modo volume controlado (VCV), com VC equivalente a $8 \mathrm{ml} / \mathrm{kg}$ do peso ideal, onda de fluxo quadrada correspondente a $30 \mathrm{l} / \mathrm{min}$, FR de 12 ipm, pausa inspiratória de dois segundos, PEEP de $5 \mathrm{cmH}_{2} \mathrm{O}$, ausência de esforço respiratório (quando necessário, foi realizada manobra de hiperventilação utilizando o ciclo manual); após obtenção da pressão de pico (Ppico) e da pressão de platô (Pplatô), foram calculados: a complacência dinâmica pela relação entre VC e diferença entre a Ppico e a PEEP; a complacência estática pela relação entre VC e a diferença entre a Pplatô e a PEEP, e a resistência das vias aéreas pela relação entre a diferença das Ppico e Pplatô pelo fluxo inspiratório realizado por meio do ventilador (Servo 
Monterey K.TAKAOKA São Paulo-Brasil). A aspiração das vias aéreas era realizada antes dessa mensuração.

Em seguida foi realizada a avaliação da força muscular inspiratória, em que o primeiro esforço deflagrado pelo paciente representou a (PI) e ao término dos 25 segundos $(23,24)$, obtendo-se o esforço máximo no qual foi determinado a $\mathrm{PI}_{\text {MÁx }}$ por meio do manovacuômetro (GER-AR ${ }^{\circledR}$, São Paulo, Brasil).

Após a avaliação inicial, que constou da análise da mecânica respiratória e da força muscular inspiratória, aqueles que preencheram os critérios de inclusão tiveram seus parâmetros de PEEP e PSV alterados para 05 e $07 \mathrm{cmH}_{2} \mathrm{O}$, respectivamente.

Concomitantemente, foi coletada amostra de sangue arterial para análise hemogasométrica por meio do gasímetro (ABL 800 Flex, Radiometer, Portugal) e monitoradas por meio do monitor multiparamétrico (DX 2010/DIXTAL-Manaus-Brasil) as seguintes variáveis: $\mathrm{SpO}_{2}$, PAS, $\mathrm{PAD}$, frequência cardíaca (FC) e FR, temperatura axilar $\left(\mathrm{T}^{\circ} \mathrm{C}\right)$. Por meio do ventilômetro (Wright MK 8 Ferraris - Reino Unido), foram obtidos volume minuto (VM) e VC. Após 30 minutos, todos os parâmetros foram reavaliados. Os pacientes que apresentaram condições para retirada da AVM e após comum acordo entre o médico e o fisioterapeuta de plantão, a conduta foi: para pacientes com uso de tubo orotraqueal, este foi removido; para aqueles que se encontravam traqueostomizados, mantinha-se a órtese ventilatória, sendo estes colocados em ventilação espontânea. No entanto, para todos que necessitaram de oxigênio suplementar, tal procedimento ficou a cargo da equipe da própria instituição.

Após realizado o desmame, esperava-se 48 horas para verificar o sucesso do paciente fora da AVM. Caso houvesse retorno para a AVM em menos de 48 horas, seria considerado insucesso.

Utilizaram-se também neste estudo ferramentas que possibilitam integração das variáveis fisiológicas, conhecidas como índices preditivos de sucesso ao desmame, as quais não foram ferramentas de predição, mas sim tidas como variáveis fisiológicas integradas. Dessa forma, também se realizou o comparativo entre elas no momento inicial e final.

\section{Análise estatística}

Para análise estatística, foram utilizados os softwares SPSS 13.0 para Windows e o Excel 2003. Todos os testes foram aplicados com 95\% de confiança.
A normalidade dos dados foi verificada pelo teste Kolmogorov-Smirnov para variáveis quantitativas. Os dados estão apresentados em tabelas com suas respectivas frequências absolutas e relativas. As variáveis quantitativas estão apresentadas por meio de medidas descritivas, como média e desvio-padrão. Para comparação entre variáveis quantitativas, foi utilizado o teste de Wilcoxon.

\section{Resultados}

Dos 17 pacientes que conseguiram concluir o protocolo, $4(17,65 \%)$ apresentaram falha ao desmame; contudo, $13(82,35 \%)$ se mantiveram fora da AVM e não retornaram ao suporte ventilatório e, por conseguinte, receberam alta da unidade coronariana.

Ao analisar as variáveis hemodinâmicas ao início e ao término do teste, verificou-se tendência à redução da FC e da PAD; todavia, sem significância estatística. Os demais dados se mantiveram praticamente inalterados, como mostra a Tabela 1.

Após análise correspondente às variáveis dos resultados da gasometria arterial, comparados entre o início e término do TRE, foi constatada uma discreta redução da pressão parcial de oxigênio no sangue arterial $\left(\mathrm{PaO}_{2}\right)$, como também do índice de troca $\left(\mathrm{PaO}_{2} / \mathrm{PAO}_{2}\right)$, mas sem significância estatística. Os demais dados estiveram praticamente inalterados, como mostra a Tabela 2.

Quando analisadas as variáveis respiratórias, verificou-se uma pequena diferença ao comparar os valores do VC, VM, PI pela $\mathrm{PI}_{\mathrm{MÁx}}$, como também os valores isolados da PI. Contudo, sem significância estatística. Os demais dados permaneceram praticamente inalterados, como mostra a Tabela 3.

Ao efetivar a comparação referente às variáveis da mecânica do sistema respiratório durante o início e o término do teste de respiração espontânea, verificou-se tendência a um discreto aumento da complacência estática, porém sem significância estatística. Os demais dados permaneceram praticamente inalterados, como mostra a Tabela 4.

No que diz respeito às alterações nas variáveis fisiológicas integradas, percebeu-se mudança significativa apenas no índice de desmame ventilatório Ferrari-Tadini (IDV), o qual integra vários parâmetros fisiológicos, como Glasgow, VC, fração inspirada de oxigênio $\left(\mathrm{FiO}_{2}\right), \mathrm{SpO}_{2}, \mathrm{PaO}_{2}, \mathrm{FR}, \mathrm{PI}_{\text {MÁX }}$ PSV, idade e por fim a $\mathrm{PaCO}_{2}$, conforme descrito na Tabela 5 . 
Tabela 1 - Comparativo das variáveis hemodinâmicas entre o início e final do TRE

\begin{tabular}{|c|c|c|c|}
\hline \multirow[b]{3}{*}{ Variáveis } & \multicolumn{2}{|c|}{ Momento } & \multirow[b]{3}{*}{$\mathrm{p}$-valor* } \\
\hline & Início & Fim & \\
\hline & Média \pm DP & Média \pm DP & \\
\hline \multicolumn{4}{|c|}{ Hemodinâmica } \\
\hline $\mathrm{PAS}(\mathrm{mmHg})$ & $134,18 \pm 19,080$ & $135,53 \pm 19,83$ & 0,421 \\
\hline $\operatorname{PAD}(\mathrm{mmHg})$ & $76,35 \pm 11,88$ & $73,82 \pm 14,26$ & 0,227 \\
\hline FC (bpm) & $90,24 \pm 14,01$ & $86,71 \pm 14,23$ & 0,114 \\
\hline
\end{tabular}

Legenda: $\mathrm{PAS}=$ Pressão arterial sistólica; $\mathrm{PAD}=$ Pressão arterial diastólica; $\mathrm{FC}=$ Frequência cardíaca; $\mathrm{DP}=$ Desvio-padrão; $\mathrm{BPM}=$ Batimentos por minuto; ${ }^{*}=$ Teste de Wilcoxon.

Fonte: Dados da pesquisa.

Tabela 2 - Comportamento das variáveis referente à gasometria arterial entre o início e o final do TRE

\begin{tabular}{|c|c|c|c|}
\hline \multirow[b]{3}{*}{ Variáveis } & \multicolumn{2}{|c|}{ Momento } & \multirow[b]{3}{*}{ p-valor } \\
\hline & Início & Fim & \\
\hline & Média \pm DP & Média \pm DP & \\
\hline \multicolumn{4}{|c|}{ Hemogasométrica } \\
\hline $\mathrm{PaO}_{2}(\mathrm{mmHg})$ & $114,66 \pm 28,091$ & $111,19 \pm 33,984$ & 0,649 \\
\hline $\mathrm{PaCO}_{2}(\mathrm{mmHg})$ & $32,06 \pm 6,781$ & $32,78 \pm 7,276$ & 0,080 \\
\hline $\mathrm{pH}$ & $7,44 \pm 0,060$ & $7,43 \pm 0,065$ & 0,082 \\
\hline $\mathrm{PaO}_{2} / \mathrm{PAO}_{2}$ & $0,71 \pm 0,259$ & $0,69 \pm 0,262$ & 0,795 \\
\hline $\mathrm{SaO}_{2}(\%)$ & $97,53 \pm 1,663$ & $97,06 \pm 1,819$ & 0,206 \\
\hline
\end{tabular}

Legenda: $\mathrm{PaO}_{2}=$ Pressão parcial de oxigênio no sangue arterial; $\mathrm{PaCO}_{2}=$ Pressão parcial de gás dióxido de carbono no sangue arterial; $\mathrm{SaO}_{2}=$ saturação arterial de oxigênio; $\mathrm{PAO}_{2}=$ Pressão de oxigênio alveolar; * $=$ Teste de Wilcoxon.

Fonte: Dados da pesquisa.

Tabela 3 - Comparação das variáveis respiratórias entre o início e o final do TRE

\begin{tabular}{|c|c|c|c|}
\hline \multirow[b]{3}{*}{ Variáveis } & \multicolumn{2}{|c|}{ Momento } & \multirow[b]{3}{*}{$\mathrm{p}$-valor» } \\
\hline & Início & Fim & \\
\hline & Média \pm DP & Média \pm DP & \\
\hline \multicolumn{4}{|l|}{ Respiratória } \\
\hline $\mathrm{VC}(\mathrm{ml})$ & $438,24 \pm 155,693$ & $414,76 \pm 155,419$ & 0,162 \\
\hline FR (irp) & $20,59 \pm 5,853$ & $21,35 \pm 5,350$ & 0,214 \\
\hline VM (I) & $8582,94 \pm 2255,733$ & $8367,65 \pm 1766,825$ & 0,381 \\
\hline PIMAX $\left(\mathrm{cmH}_{2} 0\right)$ & $-50,71 \pm 15,715$ & $-51,65 \pm 15,608$ & 0,529 \\
\hline PI/PIMAX $\left(\mathrm{cmH}_{2} \mathrm{O}\right)$ & $0,47 \pm 0,165$ & $0,52 \pm 0,124$ & 0,215 \\
\hline $\mathrm{Pl}\left(\mathrm{cmH}_{2} \mathrm{O}\right)$ & $-23,41 \pm 9,817$ & $-27,06 \pm 12,750$ & 0,336 \\
\hline
\end{tabular}

Legenda: $\mathrm{VC}=$ Volume corrente; $F R=$ Frequência respiratória; VM = Volume minuto; PIMÁX = Pressão inspiratória máxima; PI = Pressão inspiratória; * $=$ Teste de Wilcoxon.

Fonte: Dados da pesquisa. 
Tabela 4 - Comparativo referente às variáveis da mecânica respiratória entre o início e o final do TRE

\begin{tabular}{|c|c|c|c|}
\hline \multirow[b]{3}{*}{ Variáveis } & \multicolumn{2}{|c|}{ Momento } & \multirow[b]{3}{*}{ p-valor* } \\
\hline & Início & Fim & \\
\hline & Média \pm DP & Média \pm DP & \\
\hline \multicolumn{4}{|l|}{ Mecânica } \\
\hline C.din $\left(\mathrm{ml} / \mathrm{cmH}_{2} \mathrm{O}\right)$ & $21,47 \pm 4,82$ & $22,53 \pm 5,42$ & 0,362 \\
\hline $\operatorname{Raw}\left(\mathrm{cmH}_{2} \mathrm{O} / / / \mathrm{min}\right)$ & $14,47 \pm 5,46$ & $15,29 \pm 7,90$ & 0,809 \\
\hline C.est $\left(\mathrm{ml} / \mathrm{cmH}_{2} \mathrm{O}\right)$ & $36,76 \pm 17,98$ & $39,47 \pm 22,59$ & 0,813 \\
\hline
\end{tabular}

Legenda: C.din = Complacência dinâmica; C.est = Complacência estática; Raw = Resistência das vias aéreas; DP = Desvio-padrão; * $=$ Teste de Wilcoxon.

Fonte: Dados da pesquisa.

Tabela 5 - comparação entre as variáveis fisiológicas integradas nos momentos 2 minutos e 30 minutos do TRE

\begin{tabular}{|c|c|c|c|}
\hline \multirow[b]{3}{*}{ Variáveis } & \multicolumn{2}{|c|}{ Momento } & \multirow[b]{3}{*}{$\mathrm{p}$-valor* } \\
\hline & 2 minutos & 30 minutos & \\
\hline & Média \pm DP & Média \pm DP & \\
\hline \multicolumn{4}{|l|}{ Integradas } \\
\hline IDV & $28,12 \pm 1,453$ & $27,65 \pm 1,367$ & 0,011 \\
\hline IWI & $90,75 \pm 73,245$ & $91,27 \pm 91,617$ & 0,687 \\
\hline CROP & $44,21 \pm 32,252$ & $41,26 \pm 32,836$ & 0,554 \\
\hline IRRS & $52,24 \pm 22,588$ & $57,21 \pm 21,435$ & 0,124 \\
\hline
\end{tabular}

Legenda: IDV = Índice de desmame ventilatório Ferrari Tadinni; IWI = Índice integrativo de desmame; CROP = complacência dinâmica/frequência respiratória/oxigenação/pressão inspiratório máxima; IRRS = Índice de respiração rápida e superficial; * = Teste de Wilcoxon.

Fonte: Dados da pesquisa.

\section{Discussão}

O presente estudo verificou o comportamento das variáveis hemodinâmicas, hemogasométricas e respiratórias, de pacientes submetidos ao TRE utilizando a PSV durante o desmame da AVM, sendo tais valores obtidos nos momentos dois e trinta minutos.

Os pacientes submetidos ao TRE e que conseguiram completar os trinta minutos não apresentaram variação significativa nos parâmetros hemodinâmicos, mantendo-se estáveis tanto a FC, como os níveis pressóricos. Esse fato pode demonstrar que o referido teste realizado com PSV, associado à PEEP, acarreta menores repercussões e, possivelmente, melhor prognóstico.

Isso se encontra de acordo com o estudo de Epstein (6), no qual perceberam que o desmame ventilatório em pacientes cardiopatas, mais especificamente os pacientes com ICC, pode acarretar aumento do trabalho respiratório e demanda cardiovascular, sendo essas alterações atenuadas ou evitadas quando se utiliza a PSV. O mesmo estudo analisou o desmame de 31 pacientes submetidos ao TRE com tubo t por 30 minutos, evidenciando que os que não responderam a esse teste foram submetidos imediatamente a PSV e PEEP de 07 e $05 \mathrm{cmH}_{2} \mathrm{O}$ respectivamente. Desses, 21 obtiveram sucesso ao TRE, sugerindo que o tubo $t$ pode contribuir para a falha iatrogênica do desmame.

Além disso, o aumento da demanda energética pode ser considerado fator coadjuvante na condução do desmame. Santos (25) avaliou o gasto energético de 40 pacientes submetidos ao TRE e concluiu que os pacientes em uso de tubo $t$ despenderam 14,4\% a mais do que quando em PSV. Observou ainda que 
houve boa sincronia entre o ventilador e o paciente nessa modalidade, o que permitiu reduzir o esforço muscular e evitar a fadiga.

De acordo com estudos prévios, a interrupção da ventilação mecânica pode induzir várias alterações cardiovasculares, como aumento do volume diastólico final e pós-carga, arritmias, estiramento agudo dos átrios e ventrículos. Daí a importância de se avaliar tais variáveis com o propósito de evitar tais disfunções e, consequentemente, o prolongamento da AVM. Além disso, de acordo com Shen et al. (26), a variação da FC durante o TRE é uma importante ferramenta para avaliar a resposta do sistema nervoso autônomo; quando realizaram o TRE cujo método utilizado foi a mudança do PSV para o tubo t, a FC apresentou variação significativa naqueles indivíduos, cujo desmame foi mal sucedido, enquanto que, para o grupo sucesso, tal variação não ocorreu.

Chatila et al. (27), ao realizarem desmame ventilatório de 93 pacientes, obtiveram sucesso em 56 $(60,2 \%)$ dos casos; porém, insucesso em 37 (39,8\%), e destes, 6 apresentaram isquemia miocárdica, provavelmente em virtude do aumento na demanda cardiopulmonar determinada pela transição para ventilação espontânea.

Sabetzk e Cicotoste (5), quando analisaram a comparação das estratégias para o desmame em pacientes cardiopatas, verificaram que o desmame com PSV e tubo $t$, além de serem mais utilizados, representam também os métodos mais eficazes, relatando sucesso em ambos de aproximadamente 100\%.

El-Khatib e Bou-Khalil (28) relataram que o nível adequado de pressão no modo PSV difere substancialmente de paciente para paciente. Porém, ponderam que grande parte dos estudos recomenda o uso de PSV de 7 e PEEP de $5 \mathrm{cmH}_{2} \mathrm{O}$ para compensar o trabalho imposto pelo circuito/ventilador e tubo endotraqueal.

0 tempo de 30 minutos em PSV foi suficiente para que a maioria dos pacientes evoluísse para o desmame da AVM, não necessitando um tempo maior como é observado em alguns estudos. Segal et al. (29) relataram em seu estudo, em que utilizaram duas horas para a conclusão do TRE, que 17 pacientes foram extubados antes do término por decisão médica, mostrando um alto valor preditivo positivo para os dados obtidos em 30 minutos, sugerindo que este tempo pode ser suficiente para predizer a extubação bemsucedida. Boles et al. (14) também comentam que a taxa de sucesso de um teste inicial de 30 minutos em comparação com 2 horas são semelhantes.
A estabilidade hemodinâmica, hemogasométrica e respiratória, conseguida durante a realização da pesquisa mediante a utilização do TRE com PSV, vai de encontro ao estudo de Costa et al. (30), em que mostraram que, comparando o desmame realizado com PSV, houve melhora da oxigenação e dos parâmetros respiratórios demonstrado pela redução da FR, aumento do VC e VM quando comparados àqueles que utilizaram tubo t. Também verificaram valores significativamente menores de $\mathrm{FC}$, maiores para a $\mathrm{PaO}_{2}$, dióxido de carbono corrente final e $\mathrm{SaO}_{2}$ nos grupos que utilizaram a PSV.

A mensuração da $\mathrm{PI}_{\mathrm{MÁX}}$, a qual foi realizada para avaliar as variáveis respiratórias antes e imediatamente após o TRE, mostrou que os valores estão dentro dos parâmetros aceitáveis para iniciar este processo e, consequentemente, promover subsídios para continuidade da prova. Esses valores vão de encontro com o estudo de Freitas e David (13), que relatam que a $\mathrm{PI}_{\text {MÁx }}$ $<-20 \mathrm{cmH}_{2} \mathrm{O}$, antes de iniciar o desmame, pode predizer o sucesso. Isso também foi relatado por Boles et al. (14) sobre a $\mathrm{PI}_{\text {MÁX }}<-20-25 \mathrm{cmH}_{2} \mathrm{O}$ ter sido referenciada como um índice preditivo de desmame, corroborando com Caruso et al. (31), o qual descreve que a $\mathrm{PI}_{\text {MÁX }}$ é útil para quantificar o grau de força muscular respiratória em paciente no processo de desmame.

Os parâmetros respiratórios avaliados no presente estudo foram realizados em dois momentos distintos, início e fim, não mostrando diferença significativa, o que pode estar relacionado ao alto grau de sucesso ao desmame, podendo ser atribuído, entre outros fatores, à homogeneidade da amostra e à utilização da PSV, que proporcionaram adaptação e transição segura para ventilação espontânea. Não corroborando com o estudo de Conti et al. (32), em que realizaram a mensuração das variáveis VC, VM, FR e PIMÁX, em um único momento, nos primeiros dois minutos, perceberam que elas foram pobres preditores no desfecho do desmame.

Os resultados obtidos durante o TRE, referentes à estabilidade hemodinâmica, gasométrica e respiratória, corroboram ainda com os resultados do estudo de Frazier et al. (33), que avaliaram essas variáveis durante descontinuação da ventilação mecânica, utilizando o modo CPAP em 43 pacientes, sendo 28 sucessos e 15 insucessos, sendo 10 portadores de alterações cardíacas. As variáveis referentes à gasometria arterial, PAS e PAD, FC, VC não diferiram significativamente entre os grupos. De forma similar Salam et al. (34) referiram em seu estudo, que os pacientes com 
evolução satisfatória durante o TRE e que foram extubados foram justamente aqueles que mantiveram a estabilidade hemogasimétrica, hemodinâmica e respiratória. Já aqueles que não foram extubados apresentaram aumento significativo da FC e tendência ao aumento da FR e da PAM.

Apesar de não haver variação da mecânica respiratória, os valores obtidos neste estudo foram, em todos os momentos, mais baixos que aqueles preconizados como valores de normalidade relatados na literatura. Contudo, esses valores não influenciaram o resultado para o sucesso ao desmame da AVM, uma vez que, dos 17 pacientes que conseguiram passar no teste de respiração espontânea, apenas quatro evoluíram para o insucesso.

Fato semelhante foi percebido por Nozawa et al. (35), que, após submeter 45 pacientes ao desmame da AVM utilizando PSV, obtiveram 22 sucessos e 23 insucessos e perceberam que, em ambos os grupos, a maior parte apresentou valores de complacência estática abaixo da normalidade e que os dados da mecânica respiratória não interferiram no sucesso ou insucesso do desmame.

Como forma de predição do sucesso ao desmame, foram preconizados diversos índices, os quais foram obtidos mediante a utilização integrada de variáveis fisiológicas. Todavia, no corrente estudo não foi possível estabelecer critérios como sensibilidade e especificidade para tais marcadores, sendo realizada, porém, a comparação dos resultados obtidos do IRRS, CROP e IWI ao início e ao término do TER. Os valores encontrados foram condizentes com a normalidade, segundo proposto pelos seus idealizadores, com os respectivos referencias IRRS $(<105)$ e CROP $(>13$ $\mathrm{ml} / \mathrm{rpm}$ ) desenvolvidos por Yang e Tobin (36), Nemer (37) (que mostrou em seu estudo o IWI > 20).

Em relação aos índices, não houve diferença quando comparados o início e o término do TRE, com exceção do IDV, o qual mostrou resultados estatísticos significativos. Esse fato pode ter ocorrido graças a tal índice apresentar vários parâmetros para obtenção de seu resultado, mesmo a amostra sendo homogênea. Contudo, tal resultado está dentro dos parâmetros de normalidade para evolução do desmame da ventilação mecânica, que são os escores compreendidos entre 23 e 30 . De acordo com o estudo de Ferrari e Tadini (38), os resultados para a obtenção do sucesso para o desmame são classe I (27-30 indicado), classe II (23-26 favorável), classe III (20-22 desfavorável), classe IV ( $<19$ contraindicado).

\section{Considerações finais}

Por meio do presente estudo, percebeu-se que a maior parte dos pacientes cardiopatas submetidos ao teste de respiração espontânea utilizando a PSV com 7 e PEEP de $5 \mathrm{cmH} 20$ manteve estabilidade hemodinâmica, hemogasométrica e respiratória durante todo o período de avaliação. Além disso, observouse que cerca de $82 \%$ obteve sucesso ao desmame; dessa forma, esse achado possibilita aos profissionais atuantes em unidades de terapia intensiva um método seguro e eficaz na condução da retirada de paciente cardiopatas da AVM.

Também foi percebido e sustentado pela literatura que as alterações na mecânica respiratória não são fatores que impedem ou indicam o desmame da AVM, sendo mais importante a estabilidade evidenciada entre o início e o término do TRE. Contudo, por causa da amostra reduzida, a continuidade do presente estudo bem como o desenvolvimento de estudos semelhantes serão necessários.

\section{Referências}

1. Ruppel GL. Ventilação. In: Scalan CL, Wilkins RL, Stoller JK. Fundamentos da terapia intensiva de Egan. São Paulo: Manole; 2000. p. 205-25.

2. Almeida IP, Bertucci NR, Lima VP. Variações da pressão inspiratória máxima e pressão expiratória máxima a partir da capacidade residual funcional ou da capacidade pulmonar total e volume residual em indivíduos normais. 0 Mundo da Saúde. 2008;32(2):176-82.

3. de Carvalho CRR, Toufen C Junior, Franca SA. Ventilação mecânica: princípios, análise gráfica e modalidades ventilatórias. J Bras Pneumol. 2007;33(Supl 2): S54-S70. doi: 10.1590/S1806-37132007000800002.

4. Macintyre NR. Respiratory mechanics in the patient who is weaning from ventilator. Respir Care. 2005; 50(2):275-86.

5. Sabetzk SM, Cicotoste CL. Desmame ventilatório de pacientes cardíacos, internados na unidade coronariana: Comparação entre SIMV, PSV e TUBO T. Anais do III Seminário de Fisioterapia da Uniamérica. 2009 maio 25-26; Foz do Iguaçu, Paraná. [acesso 20 de março de 2010];3:1-4. Disponível em: http://www.uni america.br/transporte/3seminario/psd/artigos/ StefaniUTI.pdf. 
6. Epstein S. Weaning from ventilatory support. Curr Opin Crit Care. 2009;15(1):36-43.

7. Herlity JP, Koch SM, Jackson R, Nora H. Course of weaning from prolonged mechanical ventilation after cardiac surgery. Tex Heart Inst J. 2006;33(2):122-9.

8. Wynne R, Botti M. Postoperative pulmonary dysfunction in adults after cardiac surgerywith cardiopulmonary bypass: clinical significance and implications for practice. Am J Crit Care. 2004;13(5):384-93.

9. Pasquina P, Merlani P, Granier JM, Ricou B. Continuous positive airway pressure versus noninvasive pressure support ventilation to treat atelectasis after cardiac surgery. Anesth Analg. 2004;99(4):1001-8.

10. Weissman C. Pulmonary function after cardiac and thoracic surgery. Anesth Analg. 1999;88(6):1272-9.

11. Godwasser R, Farias A, Freitas EE, Saddy F, Amado V, Okamoto VN. Desmame e interrupção da ventilação mecânica. J Bras Pneumol. 2007;33(2):128-136. doi: 10.1590/S1806-37132007000800008.

12. Colombo T, Baldrini AF, Juliano SRR, Juliano MCR, Houly JGS, Gebara OCE, et al. Implementação, avaliação e comparação dos protocolos de desmame com tubot e pressão suporte associada à pressão expiratória final positiva em pacientes submetidos a ventilação mecânica por mais de 48 horas em unidade de terapia intensiva. Rev Bras Ter Intensiva. 2007;19(1):31-7. doi: 10.1590/S0103-507X2007000100004.

13. Freitas EEC, David CMN. Avaliação do sucesso do desmame da ventilação mecânica. Rev Bras Ter Intensiva. 2006; 18(4): 351-9. doi: 10.1590/S0103-507X 2006000400006.

14. Boles JM, Bion A, Connors M, Herridge B, Marsh C, Melot R, et al. Weaning from mechanical ventilation. Eur Respir J. 2007;29(5):1033-56.

15. Vivar FF, Ferguson ND, Esteban A, Epstein SK, Arabi Y, Apezteguía C, et al. Risk factors for extubation failure in patients following a successful spontaneous breathing trial. Chest. 2006;130(6):1664-71.

16. Koizumi MS, Araújo GL. Escala de Coma de Glasgow: subestimação em pacientes com respostas verbais impedidas. Acta Paul Enferm. 2005;18(2):136-42.

17. Teasdale G, Jennett B. Assessment of coma and impaired consciousness. Lancet. 1974;13;2(7872):81-4.
18. Chamorro CJL, Melgarb M, Barrientos R. Monitorización de la sedación. Med Intensiva. 2008;32(Suppl 1): 45-52.

19. Nascimento DBD, Nascimento JEA, Costa CBAL, Vale HV, Gava MM. Precisão de métodos de estimativa do peso e altura na avaliação do estado nutricional de pacientes com câncer. Rev Bras Nutr Clin. 2006; 21(2):111-6.

20. Brow RG, Hopkins J, Michael B, Morris A, David S, Thompson T, et al. Ventilation with lower tidal volumes as compared with traditional tidal volumes for acute lung injury and the acute respiratory distress syndrome. N Engl J Med. 2000;342(18):1301-8.

21. Juliano SRR, Juliano MCR, Cividanes JP, Houly JGS, Gebara OCE, Cividanes GVL, et al. Medidas dos níveis de pressão do balonete em unidade de terapia intensiva: considerações sobre os benefícios do treinamento. Rev Bras Ter Intensiva. 2007;19(3):317-21.

22. Jerre G, Silva TJ, Gastaldi A, Leme F, Guimarães F, Jeanette JJ, et al. III Consenso Brasileiro de Ventilação Mecânica: Fisioterapia no paciente sob ventilação mecânica. J Bras Pneumolog. 2007;33(Supl 2):S142-S50.

23. Truivit JD, Marini A. Validation of a technique to assess maximal inspiratory pressure in poorly cooperative patients. Chest. 1992;102(4):1216-9.

24. Nemer, SN. Avaliação da força muscular inspiratória (PI Máx), da atividade do centro respiratório (P 0.1) e da relação da atividade do centro respiratório/força muscular inspiratória (P 0.1/ PI Máx) sobre o desmame da ventilação mecânica [tese]. São Paulo: Faculdade de Medicina da Universidade de São Paulo; 2007.

25. Santos LJ. Avaliação do gasto energético durante o desmame da ventilação mecânica nos modos pressão suporte e tubo t. [dissertação]. Rio Grande do Sul: Universidade Federal do Rio Grande do Sul; 2008.

26. Shen HN, Lin LY, Chen KY, Kuo PH, Yu CJ, Wu HD, et al. Changes of heart rate variability during ventilator weaning. Chest. 2003;123(4):1222-8.

27. Chatila W, Ani S, Guaglianone D, Jacob B, Adjepong YA, Manthous CA. Cardiac ischemia during weaning from mechanical ventilation. Chest. 1996;109:1577-83.

28. El-Khatib MF, Bou-Khalil P. Clinical review: liberation from mechanical ventilation. Crit Care. 2008;12(4): 221. 
29. Segal LN, Oei E, Oppenheimer BW, Goldring RM, Bustami RT, Ruggiero S, et al. Evolution of pattern of breathing during a spontaneous breathing trial predicts successful extubation. Intensive Care Med. 2010;36(3):487-95.

30. Costa AD, Reider MM, Vieira SRR. Desmame da ventilação mecânica utilizando pressão de suporte ou tubo t. comparação entre pacientes cardiopatas e não cardiopatas. Arq Bra Cardiol. 2005;85(1):32-8.

31. Caruso P, Friedrich C, Denari S, Ruiz S, Deheinzelin D. The unidirectional valve is the best method to determine maximal inspiratory pressure during weaning. Chest. 1999;115(4):1096-101.

32. Conti G, Montini L, Pennisi MA, Cavaliere F, Arcangeli A, Bocci MG. A prospective, blinded evaluation of indexes proposed to predict weaning from mechanical ventilation. Intensive Care Med. 2004;30(5):830-6.

33. Frazier SK, Stone KS, Moser D, Schlanger R, Carle C, Pender L. Hemodynamic changes during discontinuation of mechanical ventilation in medical intensive care unit patients. Am J Crit Care. 2006;15(6):580-93.

34. Salam A, Smina M, Gada P, Tilluckdharry L, Upadya A, Amoateng-Adjepon Y, et al. The effect of arterial blood gas values on extubation decisions. Respir Care. 2003;48(11):1033-7.
35. Nozawa E, Kobayashi E, Matsumoto ME, Feltrim MIZ, Carmona MJC, Auler JOC Jr. Avaliação de fatores que influenciam no desmame de pacientes em ventilação mecânica prolongada após cirurgia cardíaca. Arq Bras Cardiol. 2003;80(3):301-5.

36. Yang KL, Tobin MJ. A prospective study of indexes predicting the outcome of trials of weaning from mechanical ventilation. N Engl J Med. 1991;324(21):1445-50.

37. Nemer SN, Borbas CSV, Caldeira JB, Cárias TC, Santos RG, Almeida LC, et al. A new integrative weaning index of descontinuation from mechanical ventilation. Crit Care. 2009;13(5):R152.

38. Ferrari D, Tadine R. Índice de desmame ventilatório: IDV Ferrari-tadini. [acesso 6 jul. 2009]. 2004. Disponível em: http://www.sobrati.com.br/trabalho3-jan2004.htm.
Recebido: 09/11/2010

Received: 11/09/2010

Aprovado: 23/05/2011

Approved: 05/23/2011 